\title{
Total Synthesis of Panepophenanthrin.
}

John. E. Moses, Laurent Commeiras, Jack E. Baldwin* and Robert M. Adlington.

Dyson Perrins Laboratory, Oxford University, South Parks Road, Oxford OX1 3QY, U.K.

\section{Supporting information.}

\section{General experimental details:}

All solvents and reagents were purified by standard techniques reported in Perrin, D.D.; Amarego, W. L. F., Purification of Laboratory Chemicals, $3^{\text {rd }}$ edition, Pergamon Press, Oxford, 1988 or used as supplied from commercial sources as appropriate. Solvents were removed under reduced pressure using a Buchi R110 or R114 Rotavapor fitted with a water or dry ice condenser as necessary. Final traces of solvent were removed from samples using an Edwards E2M5 high vacuum pump with pressures below $2 \mathrm{mmHg}$.

All experiments were carried out under inert atmosphere unless otherwise stated.

${ }^{1} \mathrm{H}$ NMR spectra were recorded at 400 and $500 \mathrm{MHz}$ using, Bruker DPX400, Bruker AM500 and Bruker AMX500 instruments. For ${ }^{1} \mathrm{H}$ spectra recorded in $\mathrm{CDCl}_{3}, \mathrm{CD}_{3} \mathrm{OD}$ , chemical shifts are quoted in parts per million (ppm) and are referenced to the residual solvent peak. The following abbreviations are used: s, singlet; d, doublet; $t$, triplet; m, multiplet; br, broad. Proton assignments are supported by ${ }^{1} \mathrm{H}-{ }^{1} \mathrm{H}$ COSY where necessary. Data are reported in the following manner: chemical shift (integration, multiplicity, coupling constant if appropriate). Coupling constants $(J)$ are reported in Hertz to the nearest $0.5 \mathrm{~Hz}$.

${ }^{13} \mathrm{C}$ NMR spectra were recorded at 100.6 and $125.8 \mathrm{MHz}$ using Bruker DPX400, Bruker AM500 and Bruker AMX500 instruments. Carbon spectra assignments are supported by DEPT-135 spectra, ${ }^{13} \mathrm{C}^{1}{ }^{1} \mathrm{H}$ (HMQC and HMBC) correlations where necessary. Chemical shifts are quoted in $\mathrm{ppm}$ and are referenced to the appropriate residual solvent peak.

Flash column chromatography was carried out using Sorbsil ${ }^{\mathrm{TM}}$ C60 (40-63 mm, 23040 mesh) silica gel or Florisil (100-200 mesh). Thin layer chromatography was carried out on glass plates pre-coated with Merck silica gel $60 \mathrm{~F}_{254}$ which were visualised by quenching of u.v. fluorescence or by staining with $10 \% \mathrm{w} / \mathrm{v}$ ammonium molybdate in $2 \mathrm{M}$ sulphuric acid or $1 \% \mathrm{w} / \mathrm{v}$ potassium permanganate in aqueous alkaline solution followed by heat, as appropriate.

Melting points were recorded using a Cambridge Instruments Gallen ${ }^{\mathrm{TM}}$ III Kofler Block melting apparatus or a Buchi 510 capillary apparatus and are uncorrected.

Infrared spectra were recorded either as a thin film between $\mathrm{NaCl}$ plates or as a $\mathrm{KBr}$ disc (as indicated) on a Perkin-Elmer Paragon 1000 Fourier Transform spectrometer with internal referencing. Absorption maxima are reported in wavenumbers $\left(\mathrm{cm}^{-1}\right)$ and the following abbreviations are used: w, weak; m, medium; s, strong; br, broad.

Low resolution mass spectra were recorded on V. G. Micromass ZAB $1 F$ and V. G. Masslab instruments as appropriate with modes of ionisation being indicated as CI, 
EI, ES or APCI with only molecular ions, molecular ion fragments and major peaks being reported. High resolution mass spectrometry was measured on a Waters 2790Micromass LCT electrospray ionisation mass spectrometer and on a VG autospec chemical ionisation mass spectrometer.

\section{Experimental Procedures.}

\section{TES-Protected Dimer ( )-8.}

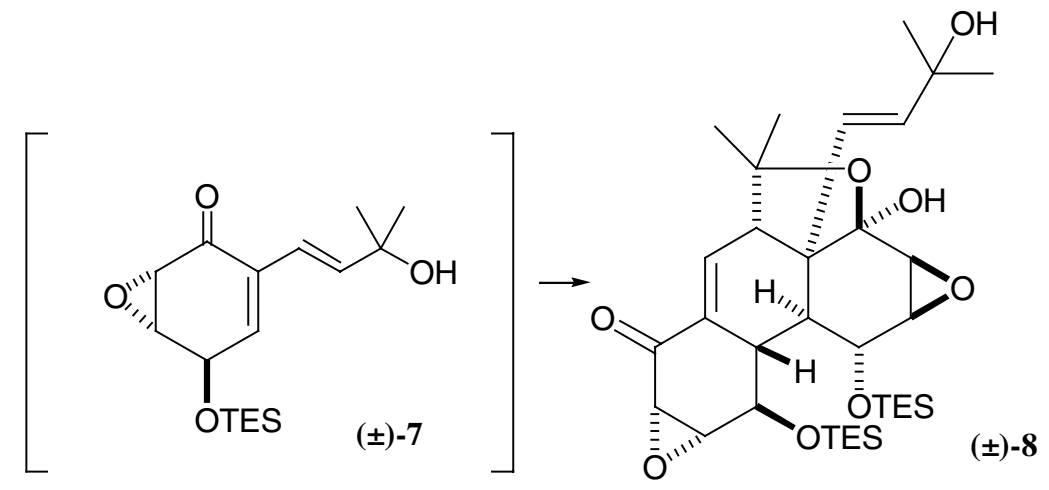

To a round-bottomed flask fitted with a water condenser was added bromoxone ( )$\mathbf{6}^{\mathbf{1}}(1.1 \mathrm{~g}, 3.4 \mathrm{mmol})$ dissolved in $20 \mathrm{~mL}$ of degassed toluene. Vinyl stannane $\mathbf{4}^{2}(1.60 \mathrm{~g}$, $4.3 \mathrm{mmol}$,) was added and the mixture was heated to $110^{\circ} \mathrm{C}$. In a separate flask, $\mathrm{Pd}_{2} \mathrm{dba}_{3}$ (320mg, $0.34 \mathrm{mmol}$ ), and $\mathrm{AsPh}_{3}(330 \mathrm{mg}, 1.08 \mathrm{mmol})$ were stirred in $10 \mathrm{~mL}$ of degassed toluene for 20 minutes. The catalyst solution was then added to the above reaction drop-wise over 10 minutes and the reaction mixture was stirred for an additional $1 \mathrm{~h}$ at $110^{\circ} \mathrm{C}$. The solvent was removed by rotary evaporation. The crude product was subjected to silica gel chromatography (1\% methanol in Chloroform) to afford two products: the monomer ( )-7 and the dimer ( )-8. But upon standing overnight, ( )-7 was transformed into ( )-8. Finally ( )-8 was obtained in 75\% overall yield (827 $\mathrm{mg})$ as a white powder.

Data for ( )-7: ${ }^{1} \mathrm{H}$ NMR (400 MHz, MeOD) $\delta_{\mathrm{H}} 0.63(6 \mathrm{H}, \mathrm{q}, J 8.0), 0.98(9 \mathrm{H}, \mathrm{t}, J 8.0)$, $1.30(3 \mathrm{H}$, br s), 1.31 (3H, br s), 3.49 (1H, br dd, J 3.7, 1.1), $3.74(1 \mathrm{H}, \mathrm{m}, J$ 3.7, 1.1, 2.5), 4.62 (1H, br d, J 5.0), 6.32 (1H, d, J 16.2), 6.44 (1H, d, J 16.2), 6.63 (1H, br dd, $J$ 5.0, 2.5); ${ }^{13} \mathrm{C}(\mathrm{MeOD}, 100.6 \mathrm{MHz}) \delta_{\mathrm{C}} 4.8,7.0,28.70,28.74,55.2,58.8,64.1,71.4$, 120.7, 134.3, 139.7, 143.4, 195.2.

\footnotetext{
${ }^{1}$ Block, O.; Klein, G.; Altenbach. H.; Brauer, D. J. J. Org. Chem. 2000, 65, 716.

${ }^{2}$ Zhang, H. X.; Guibé, F.; Balavoine, G. J. Org. Chem. 1990, 55, 1857.
} 
Data for ( )-8: $\mathrm{mp}=90{ }^{\circ} \mathrm{C} ; v_{\max } / \mathrm{cm}^{-1}(\mathrm{KBr}) 3435,1691,1599 ;{ }^{1} \mathrm{H}$ NMR $(500 \mathrm{MHz}$, $\left.\mathrm{CDCl}_{3}\right) \delta_{\mathrm{H}} 0.66(12 \mathrm{H}, \mathrm{q}, J 7.9), 0.99(9 \mathrm{H}, \mathrm{t}, J 7.9), 1.00(9 \mathrm{H}, \mathrm{t}, J$ 7.9), $1.19(3 \mathrm{H}, \mathrm{s})$, $1.23(3 \mathrm{H}, \mathrm{s}), 1.37(3 \mathrm{H}, \mathrm{s}), 1.44(3 \mathrm{H}, \mathrm{s}), 2.10(1 \mathrm{H}, \mathrm{d}, J 11.8), 2.53$ (1H, br d, $J 11.8)$, $3.29(1 \mathrm{H}$, br s), 3.41-3.46 (3H, m), $3.47(1 \mathrm{H}, \mathrm{d}, J 4.5), 3.73(1 \mathrm{H}, \mathrm{br} \mathrm{t}, J 4.5), 4.52(1 \mathrm{H}$, d, $J$ 3.5), $4.58(1 \mathrm{H}, \mathrm{d}, J 3.5), 5.68(1 \mathrm{H}, \mathrm{d}, J 16.5), 6.13(1 \mathrm{H}, \mathrm{d}, J 16.5), 6.81(1 \mathrm{H}, \mathrm{dd}, J$ 5.6, 2.5); ${ }^{13} \mathrm{C}\left(\mathrm{CDCl}_{3}, 125.7 \mathrm{MHz}\right) \delta_{\mathrm{C}} 4.9,5.1,6.8,6.9,25.5,29.6,30.2,32.2,48.3$, 49.6, 54.5, 55.3, 55.4, 56.0, 56.2, 59.6, 65.7, 68.7, 71.3, 78.6, 101.4, 129.6, 138.5, 138.7, 141.9, 194.9; m/z (ES-) 647 (100\%, M-1); HRMS (ES-) Calculated for $\mathrm{C}_{34} \mathrm{H}_{55} \mathrm{O}_{8} \mathrm{Si}_{2}\left([\mathrm{M}-\mathrm{H}]^{-}\right)$: 647.3457. Found: 647.3436.

Panepophenanthrin ( )-1. ${ }^{3}$

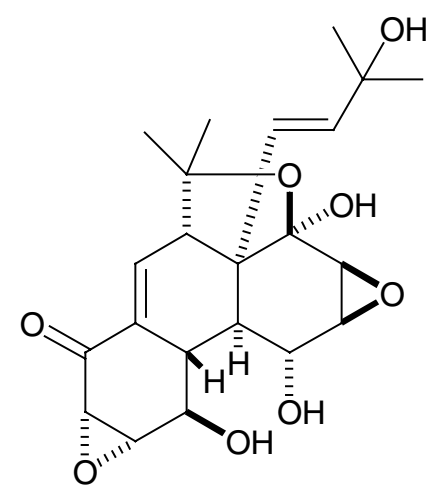

Panepophenanthrin $( \pm)-1$

To a $25 \mathrm{~mL}$ round-bottomed flask containing methanol $(5 \mathrm{~mL})$, was added ( )-8 (199mg, $0.31 \mathrm{mmol}$ ), the solution was allowed to stir for 5 minutes. $\mathrm{NH}_{4} \mathrm{~F}(45.5 \mathrm{mg}$, $1.23 \mathrm{mmol})$ was then added and the mixture allowed to stir until the reaction had completed (approx 2h). The solvent was removed under reduced pressure and the crude mixture subjected to silica gel chromatography (3\% methanol in chloroform) to give the title compound as a white solid $(110 \mathrm{mg}, 85 \%) \cdot \mathrm{Rf}=0.3\left(9: 1 \mathrm{CHCl}_{3}: \mathrm{MeOH}\right)$; $\mathrm{mp}=81{ }^{\circ} \mathrm{C} ; v_{\max } / \mathrm{cm}^{-1}(\mathrm{KBr}) 3435,1672,1600 ;{ }^{1} \mathrm{H}$ NMR (500 MHz, MeOD) $\delta_{\mathrm{H}} 1.17$ $(3 \mathrm{H}, \mathrm{s}), 1.20(3 \mathrm{H}, \mathrm{s}), 1.35(3 \mathrm{H}, \mathrm{s}), 1.45(3 \mathrm{H}, \mathrm{s}), 2.04(1 \mathrm{H}, \mathrm{br} \mathrm{d}, J 10.0), 2.34(1 \mathrm{H}, \mathrm{m}, J$ 10.0, 3.0, 2.0), 3.32 (1H, d, J 4.1), 3.35 (1H, dd, J 5.1, 2.0), 3.42 (1H, d, J 4.1), 3.50 (1H, br t, J 3.5), $3.83(1 \mathrm{H}$, br t, $J 3.5), 4.35$ (1H, br t, $J 2.2), 4.55(1 \mathrm{H}$, br t,$J 2.2), 5.68$ $(1 \mathrm{H}, \mathrm{d}, J 16.2), 5.98(1 \mathrm{H}, \mathrm{d}, J 16.2), 6.82(1 \mathrm{H}, \mathrm{dd}, J 5.1,3.0) ;{ }^{13} \mathrm{C}(\mathrm{MeOD}, 125.7$

\footnotetext{
${ }^{3}$ Sekizawa, R.; Ikeno, S.; Nakamura, H.; Naganawa, H.; Matsui, S.; Iinuma, H.; Takeuchi, T. J. Nat. Prod. 2002, 65, 1491.
} 
MHz) $\delta_{\mathrm{C}} 26.2,29.5,30.3,32.3,50.0,51.2,55.1,55.6,57.1,57.2,57.3,60.7,66.3$, 69.0, 71.8, 79.2, 102.6, 129.2, 138.8, 139.9, 143.1, 196.2; HRMS (ES-) Calculated for $\mathrm{C}_{22} \mathrm{H}_{27} \mathrm{O}_{8}\left([\mathrm{M}-\mathrm{H}]^{-}\right)$: 419.1704. Found: 419.1706.

\section{Comparison of the ${ }^{1} \mathrm{H}$ NMR of Natural and Synthetic Panepophenanthrin 1.}

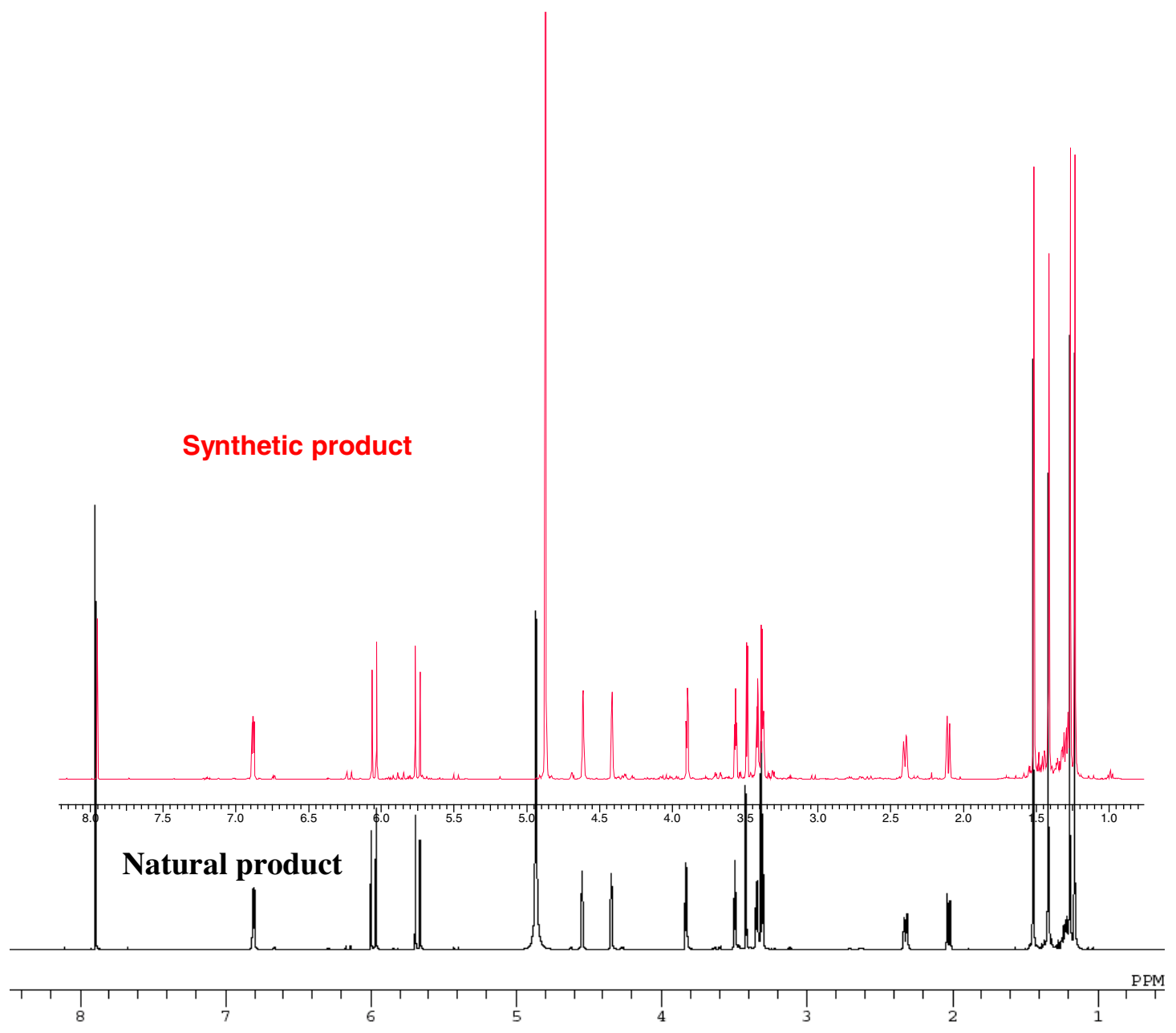


Comparison of the ${ }^{13} \mathrm{C}$ NMR of Natural and Synthetic Panepophenanthrin 1.

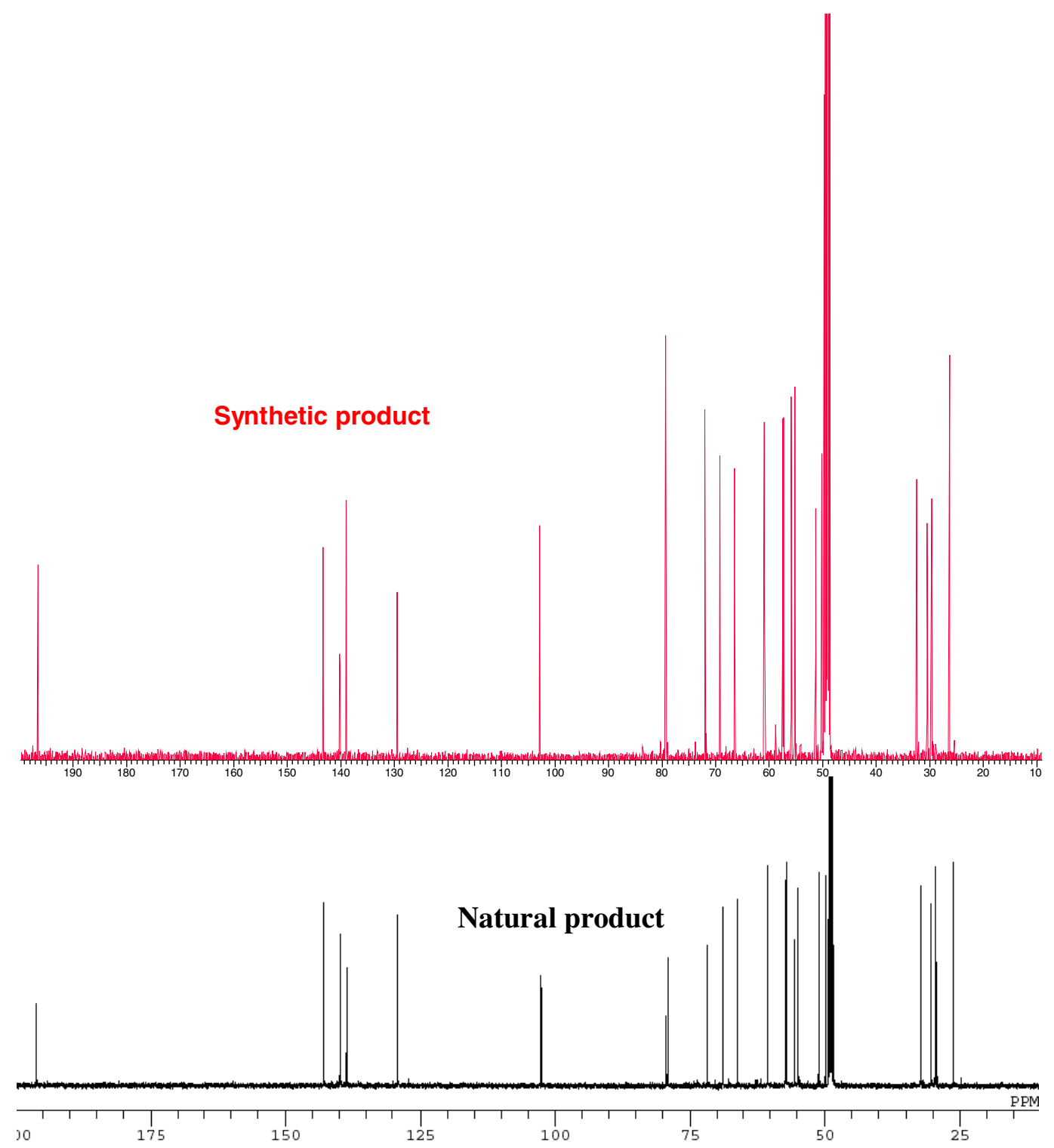

